\title{
Factors associated with the safety skills of patients receiving bDMARDs in chronic rheumatic diseases
}

\author{
B. Bengana, A.Boukabous,N. Bahaz , S. Mostefai , S. Lefkir tafiani \\ Derpartement of Rheumatology -University hospital of Beni Messous, University of Algiers, Algiers, \\ Algeria
}

\section{Introduction}

Biologics have become increasingly common in the treatment of inflammatory rheumatic diseases. As these drugs are more widely prescribed, healthcare providers need to be more educated about everything from the effectiveness and safety of biologics to how to properly administer the medication. We aimed to determine patient factors associated with the safety skills of patients receiving bDMARDs for inflammatory arthritis.

\section{Results}

We have asked 121 patients with mean age $43 \pm 11$ years old; 52 (43\%) women; indications for biologics were mainly rheumatoid arthritis in 51 cases, anklyosing spondylitis in 37cases, 21 or enteropathic arthritis and 12 for psoriatic arthritis ; 88 (73\%) received subcutaneous bDMARDs. Patients had received information about their treatments from their physician 97 (80\%), a nurse 6 (5\%), by a written booklet 24 (20\%), and / or during therapeutic patient education (TPE) sessions $12(10 \%)$. In total, 28 $(23,4 \%)$ patients had a low skill level; $74(61.2 \%)$ a moderate skill level and $19(15.4 \%)$ a high skill level. On multivariate regression analysis, as compared with high safety skills, low skills were associated with living in rural areas (OR $2.9\left[\begin{array}{lll}95 \% & \mathrm{Cl}\end{array}\right]$, low educational level (OR 3.8 ), living in a city (OR 3.2), and not participating in TPE sessions or consulting a nurse (OR 4.3 ).

\begin{tabular}{lll}
\hline & N & $\%$ \\
\hline Sex & & \\
\hline Women & 76 & 56 \\
$>$ Men & 59 & 44 \\
Married women & & \\
\hline Non menopausal & 56 & 74 \\
$>$ Menopausal & 20 & 26 \\
Social status & & \\
\hline Married (s) & 83 & 61 \\
singles & 48 & 36 \\
Other & 4 & 3 \\
& & \\
Profession & 50 & 38 \\
\hline No occupation & 49 & 37 \\
Public function & 19 & 14 \\
Student-Trainee & 8 & 5 \\
trader & 5 & 4 \\
Retirement & 3 & 2 \\
Artisan & & \\
&
\end{tabular}

\section{Materials/methods}

We conducted a cross-sectional survey performed in 2016 . A questionnaire was elaborated to measure knowledge and skills regarding safety issues, for patients treated by biologics.

Table 2: Level of information in patients on the risk of taking bDMARDs

\section{Some interesting questions \\ N $\%$}

about safety skills of our

\section{patients}

State of the patient before beginning biotherapy

No hesitation

Refusal at departure

Short time of reflection

Long time of reflection

$\begin{array}{ll}71 & 79 \\ 14 & 16 \\ 3 & 3 \\ 2 & 2\end{array}$

Informed consent before

biotherapy

73

YES

98

27

NO

Has the doctor provided information on the risks of biotherapy?

YES

NO 109

YES but I was left with questions.

2

Do you know that the risk of

infection is higher after trt?

$\begin{array}{lll}\text { YES } & 68 & 50.4 \\ \text { NO } & 67 & 49.6\end{array}$

Do you know that the risk of neoplasia is higher after trt? YES 51 66 49

\section{Conclusion}

Biologics are known to entail some specific risks. Patients should possess self-care safety skills to develop appropriate behaviors in situations of risks. Through our study we have detected some factors associated with low safety skills of patients receiving bDMARDs, which should be addressed to improve safety skills in this population. 